\title{
The economic and human costs of asthma in Scotland
}

\author{
Ronald G Neville, Gaylor Hoskins, Barbara Smith, Colin McCowan
}

\begin{abstract}
Aim: To review the human and economic cost of asthma and its impact on the Health Service.

Methods: Observational study of 9467 patients of all ages and clinical severity of asthma in 319 Scottish general practices. The 'human cost' was calculated from assessment of symptoms and lifestyle disruption. Direct health care costs were determined from study of health service utilisation.

Results: Only $1 / 3$ of patients were free of asthma symptoms when reviewed and $770(12 \%)$ reported lost time from work/school due to asthma in the past month. 1916(20\%) patients experienced an acute exacerbation in the previous 12 months.
\end{abstract}

1,412(15\%) patients were on medication step zero of the British Thoracic Society (BTS) guidelines, 1660(18\%) step one, 4310(45.5\%) step-two, 1386(15\%) step-three, 594(6\%) step-four, and $105(1 \%)$ step five. The major component of health service cost was maintenance medication (67\% of total). Only $5 \%$ of patients received hospital treatment but accounted for $19 \%$ of total direct health care spend.

Conclusions: The human and economic costs of asthma are substantial and highlight a need to achieve improved clinical outcome. The simplistic data collection markers in the New GP Contract are unlikely to promote favourable change in outcome.

Key words: Asthma, economics, symptoms, lifestyle, New GP Contract

\section{Ronald G Neville}

Asthma Research Unit,

University of Dundee

\section{Gaylor Hoskins}

Asthma Research Unit,

University of Dundee

\section{Barbara Smith}

Asthma Research Unit,

University of Dundee

\section{Colin McCowan}

Asthma Research Unit, University of Dundee

\section{Correspondence to:} Mrs Gaylor Hoskins Asthma Research Unit Tayside Centre for General Practice Kirsty Semple Way Dundee DD2 4BF

\section{Email:}

g.hoskins@dundee.ac.uk

Date submitted:22/10//03

Date accepted: 17/11/03

Prim Care Resp J 2003; 12(4)115-119

\section{Introduction}

Asthma is a major cause of morbidity in all countries of the world, including Scotland. ${ }^{1}$ Despite equitable access to a free system of health care and the availability of modern and effective treatments asthma is the leading cause of medical admission in childhood, disrupts schooling, leads to days lost from work and impairs the quality of life of many people of all ages. A recent report from the National Asthma Campaign in Scotland highlighted the impact asthma has on individuals, the health service and society. ${ }^{2}$

In the 1990s a quiet revolution took place in the way asthma was diagnosed and managed within primary care. ${ }^{3}$ Many practices organised dedicated asthma clinics run by practice nurses with specific training. ${ }^{4}$ Closer ties between primary and secondary care promoted a shared understanding of the need to manage asthma as a chronic condition. ${ }^{5}$ The widespread use of inhaled steroids together with educational initiatives including guided self management plans was underpinned by the publication of the British Thoracic Society (BTS) guidelines. ${ }^{6}$

The management of asthma has important cost implications. Direct health service costs include medications, primary care consultations, hospital accident and emergency and outpatient attendance and hospital admissions. Indirect costs are those applicable to individual patients, their families, and lost opportunities for work or education. ${ }^{7}$ In an era of quality assurance initiatives, guidelines, clinical governance and cost containment it is pertinent to review how an individual medical condition impacts on the lives of sufferers and on all tiers of the health service. Health service planning and patient focused interventions are hampered by a lack of national information from a patient and a health service perspective.
There is a misperception amongst some clinicians and health service providers that the 'asthma problem' has been resolved. The widespread availability of practice asthma clinics, increased use of appropriate medication and a recent modest reduction in admission rates has led to complacency. Asthma can and does kill people and the 'asthma problem' is anything but resolved. The New GP Contract features asthma as one of 10 important long-term conditions. Practices may be able to earn 72 'quality points' by complying with a data collection exercise involving a register of patients, confirmation of diagnosis, record of smoking status, influenza vaccinations and clinical review (details unspecified). It is thus pertinent to review the current state of the management of asthma within Scotland in the light of shifting health service priorities, the New Contract, economic pressures, changing public and health professional perceptions, and the electronic age of communication. This paper is thus an attempt to look at the human and economic costs of one condition in one country - asthma in Scotland. This is intended to act as a benchmark against which future changes in the organisation and delivery of care can be measured.

\section{Method}

A steering group was convened with representation from general practice (GP), practice nursing (PN), Respiratory Medicine, Child Health, the National Asthma Campaign Scotland and Government. Currently available audit tools were reviewed and a set of customised audit materials suitable for the review of patients with asthma constructed. These were developed from a series of regional and national project tools designed to provide patient specific feedback to health care professionals based on the British Thoracic Society and Scottish Inter-Collegiate Guideline Network (SIGN) guidelines. ${ }^{8,9}$

A Distance Learning Programme was constructed around the audit materials and this was accredited for 
GP Post Graduate Educational Allowance (PGEA) and a Practice Nurse certificate. ${ }^{10}$ Twelve out of Scotland's fifteen Health Boards agreed that practices that completed the audit could use this to satisfy Chronic Disease Management (CDM) payments and thus save on bureaucracy.

We wrote to all 1,039 Scottish general practices inviting them to consider taking part in a national audit of asthma in Scotland. We explained the purpose of the project, the audit service on offer, and the incentives to participate and complete a full cycle of audit. We offered practices a choice of paper based or electronic recording materials.

Practices who expressed an interest were sent a set of project materials. We asked them to review 30 patients drawn from their practice register of patients with asthma using a random numbers grid supplied by the project team. This review was to be conducted using a structured clinical assessment known as the Tayside Asthma Stamp (manual or electronic version). ${ }^{8}$ The stamp prompts clinicians to ask about and record a night time, day time and activity symptom score based on the past month. 'Days lost' from work/school/normal activity in the past month should then be noted.

Practices were then asked to record primary and secondary care health service utilisation noted in medical case records, and all anti-asthma medication used currently and in the past 12 months. A telephone help line and practice visits were offered to resolve any queries. It was made very clear to practices that the project was a major commitment involving at least two full days of health professional time to see and assess patients, complete the project booklets or disks, and to receive and discuss feedback.

Practices that opted to participate completed the health service resource questionnaire, reviewed their patients (by the GP or practice nurse) and then returned completed booklets or disks to the research unit. The management of every patient was compared to the BTS/SIGN asthma guidelines. Comments were generated on the areas where guidelines were adhered to, or where management differed from the recommendations. ${ }^{11,12}$ The research unit collated practice and patient details across the entire national sample in relation to health service contacts in primary and secondary care and prescriptions for antiasthma medication. Each item of direct cost to the health service was itemised using a validated formula. ${ }^{13}$ The impact of asthma on individual patients was determined from analysis of symptom scores and 'days lost' from work/school/normal activity.

\section{Results}

\section{Practices}

Seven hundred and seventy six (74\%) of Scotland's GP practices expressed an initial interest and were thus sent project details and given an opportunity to participate. Three hundred and nineteen (30\%) enrolled and completed at least one full year of clinical reviews on their patients, sending of data and receipt of feedback. Participating practices were drawn from every Health Board area of Scotland.

Partnership size varied from single handed to seven or more partners and had a distribution not dissimilar from national patterns. Two hundred and eight (65\%) of participating practices already had approval from their local Health Board to run an Asthma Chronic Disease Management service, 232 (73\%) operated a nurse run asthma clinic, and 192 (60\%) employed a practice nurse in possession of a recognised accredited asthma diploma.

Patients

A total of 9,467 patients were recruited to the project by their practices. There were $283(3 \%)$ aged under 4, 2,292 (24\%) aged 5-15, 3,706 (39.5\%) aged 16-44, 2762 (29\%) aged 45-74 and 424 (4.5\%) aged 75 and over. There were 4,641 (49\%) males. There were 1,557 (16\%) smokers spread evenly across all adult age ranges.

Human cost: symptoms and 'days lost' Six thousand four hundred and eight (68\%) patients attended their own practice for a review of asthma in connection with the project, of whom 4211 (66\%) had experienced symptoms due to asthma in the past month. One hundred and fifteen (55\%) young people aged 0-4 had experienced cough, wheeze or

breathlessness at night, during the day and on activity within the past month. Daytime symptoms were reported by $56 \%$ of adults aged $16-44,62 \%$ aged $45-$ 74 , and $62 \%$ aged 75 plus. Activity symptoms were common in all ages with the prevalence rising to 53\% in patient aged 75 and above. Table 1 reports the relationship between symptom score and age in more detail.

Thirty six (17\%) children aged 0-4 and 165 (10\%) of those aged 5-15 lost days from normal activity due to asthma in the past month. Lost days were reported in $11 \%$ of patients in the $16-44$ age band, and in $14 \%$ of patients in the 45-64 and 75+ age groups.

Direct costs: Primary Care Consultations In the 12 month period prior to clinical assessment 4,097 (43\%) patients initiated 8,728 consultations with a general practitioner and 924 (10\%) went to see their practice nurse on a total of 1,462 occasions in connection with asthma. In the same time period the practices themselves initiated 2,947 routine clinical reviews of 1,693 (18\%) patients with the GP, and

Table 1 Patient symptom scores broken down by ages

\begin{tabular}{lrrrrrrr} 
& & & & & & \\
& $0-4(\%)$ & $5-15(\%)$ & $16-44(\%)$ & $45-74(\%)$ & $75+(\%)$ & $\begin{array}{r}\text { Total } \\
\text { n=208 }\end{array}$ \\
& $\mathrm{n}=1575$ & $\mathrm{n}=2120$ & $\mathrm{n}=2158$ & $\mathrm{n}=347$ & $\mathrm{n}=6408$ \\
\hline Symptom scores & & & & & & \\
No symptoms & $93(45)$ & $724(46)$ & $686(32)$ & $603(28)$ & $91(26)$ & $2197(34)$ \\
Night time symptoms & $89(43)$ & $407(26)$ & $784(37)$ & $759(35)$ & $90(26)$ & $2129(33)$ \\
Day time symptoms & $93(45)$ & $633(40)$ & $1186(56)$ & $1333(62)$ & $216(62)$ & $3461(54)$ \\
Activity symptoms & $77(37)$ & $578(37)$ & $883(42)$ & $1068(49)$ & $185(53)$ & $2791(44)$
\end{tabular}


Table 2. Asthma Consultations

$$
\begin{aligned}
& \text { 0-4 5-15 16-44 45-74 75+ Total } \\
& (n=283)(n=2292)(n=3706)(n=2762) \quad(n=424)(n=9467)
\end{aligned}
$$

\begin{tabular}{llrrrrrrl}
\hline \multicolumn{2}{l}{ Asthma review consultations } & & & & & & & \\
Nurse & Number of patients & 153 & 1179 & 1358 & 1324 & 226 & 4240 & \\
& Number of consultations & 307 & 2162 & 2159 & 2475 & 487 & 7590 & 68,310 \\
GP & Number of patients & 77 & 394 & 540 & 579 & 103 & 1693 & \\
& Number of consultations & 163 & 598 & 806 & 1190 & 190 & 2947 & 53,046 \\
Urgent asthma consultations & & & & & & & \\
Nurse & Number of patients & 45 & 258 & 267 & 316 & 38 & 924 & \\
& Number of consultations & 73 & 385 & 402 & 532 & 70 & 1462 & 13,158 \\
GP $\quad$ Number of patients & 205 & 997 & 1385 & 1310 & 200 & 4097 & \\
& Number of consultations & 653 & 1876 & 2581 & 3141 & 477 & 8728 & 157,104
\end{tabular}

Table 3. Attendances

\begin{tabular}{lrrrrrr} 
Table 3. Attendances & \multicolumn{7}{c}{ Age Group } \\
& $0-4$ & $5-15$ & $\begin{array}{r}16-44 \\
45-74\end{array}$ & $\begin{array}{r}75+ \\
(\mathrm{n}=238)\end{array}$ \\
& & $(\mathrm{n}=2292)$ & $\begin{array}{r}\text { Total } \\
(\mathrm{n}=3706)\end{array}$ \\
$\begin{array}{l}\text { Accident and Emergency } \\
\text { Number of Patients }\end{array}$ & $39(14 \%)$ & $85(4 \%)$ & $115(3 \%)$ & $72(3 \%)$ & $10(2 \%)$ & $321(3 \%)$ \\
Number of attendances & 55 & 114 & 152 & 99 & 10 & 430 \\
Outpatients & & & & & & \\
Number of patients & $44(16 \%)$ & $93(4 \%)$ & $110(3 \%)$ & $191(7 \%)$ & $17(4 \%)$ & $455(5 \%)$ \\
Number of attendances & 87 & 221 & 239 & 455 & 27 & 1029 \\
Admissions & & & & & & \\
Number of patients & $29(10 \%)$ & $47(2 \%)$ & $70(2 \%)$ & $80(3 \%)$ & $11(3 \%)$ & $237(3 \%)$ \\
Number of admissions & 38 & 63 & 87 & 123 & 12 & 323 \\
Total in-patient days & 74 & 119 & 284 & 679 & 60 & 1216 \\
Total intensive care days & 1 & 4 & 10 & 13 & - & 28 \\
& & & & & &
\end{tabular}

7,590 reviews of 4,240 (45\%) patients with the practice nurse (see Table 2).

The average cost per patient of a patient initiated GP consultation is $£ 16.59$ giving a total cost for 9,467 patients of $£ 157,104$ (£157K). The corresponding costs of patient initiated nurse consultations were $£ 1.39$ giving a total cost of $£ 13 \mathrm{~K}$. The average cost per patient of a practice initiated clinical review was $£ 5.60$ for the GP and $£ 7.22$ for the nurse giving totals of $£ 53 \mathrm{~K}$ and $£ 68 \mathrm{~K}$ respectively.

Acute Medication use

In total 3,486 episodes of acute asthma exacerbation were reported from 1,916 patients ( $20 \%$ of the total sample). In the course of these exacerbations 2,209 courses of systemic steroids were prescribed and 857 emergency nebulisations carried out. The average cost of acute medication per patient was $£ 0.71$.

\section{Maintenance Medication use}

There were 1,412 (15\%) patients reported to be on no regular anti-asthma medication, the so called 'Step 0'. At step 1 of the BTS Guidelines (bronchodilator only) there were 1,660 (18\%), Step 2 (low dose inhaled steroids or cromoglycate) 4,310 (45.5\%), Step 3 (high dose inhaled steroid therapy, or low or medium dose inhaled steroid therapy plus additional therapy) 1,386 (14.5\%), Step 4 (high dose inhaled steroid therapy with additional therapy) 594 (6\%), Step 5
Cost

(maintenance oral steroids) 105 (1\%).

Hospital service utilisation

Over the course of the previous 12 months 321 (3\%) patients had attended an Accident and Emergency (A\&E) department because of an asthma related problem, 455 (5\%) had attended out patients, and 237 (2.5\%) were admitted to hospital. Fifteen of these patients experienced a total of 28 days stay in an intensive care unit. Twelve percent of A\&E attendance, $10 \%$ of outpatient contacts and $12 \%$ of admissions were in children aged 0-4, who represented only $3 \%$ of the total patient sample [See Table 3].

Prescribed medication for maintenance treatment accounted for a total cost of $£ 1,451,535$ (£1,452K) representing $67 \%$ of the total health care spend on asthma amongst the 9,467 patients studied. Hospital costs accounted for £421K (19\%) with admissions (£306K) being the dominant component. Emergency medication represented only $0.3 \%$ of total costs.

\section{Discussion}

The 'asthma problem' is far from being solved in Scotland. Throughout the country every night and every day thousands of people with asthma experienced unpleasant symptoms. Inability to do activities affected the day to day lives of all ages from infants to the elderly. The substantial number of people recording 'days lost' due to asthma in the past month represents a burden on individuals, families and society. The 'human cost' of asthma remains high. The findings of this study, collected via health care professionals, compliments that of the patient derived findings from the National Asthma Campaign Scotland. ${ }^{2}$ There would appear to be a mis-match between New GP Contract 'quality measures' for asthma and what practices are actually doing to improve the care of patients with asthma, according to guidelines.

The results show that the management of asthma was associated with substantial costs to the National Health Service in Scotland. Prescribed preventative medication including inhaled steroids is the major component of this. Arguably, this is a favourable sign indicative of greater use of recommended guidelines. Primary care costs associated with the management of asthma are considerable. The major component of this cost was unscheduled consultations with the GP. Practices may wish to encourage greater use of practice nurses in the management of patients with asthma. This option would appear to be cheaper and evidence suggests it may be more effective.,13,14

The project was a national initiative supported and funded by Government and backed by 12 of 
Scotland's 15 Health Boards. The project demanded a substantial commitment of time and effort from participating practices, with only indirect measures taken to re-numerate practice staff through professional incentives such as PGEA and Chronic Disease Management payments. Far from being disappointed in a 30\% audit completion rate we were pleasantly surprised that 319 practice teams in Scotland were willing to invest so much of their time and energy into clinical audit. We are unaware of any other voluntary audit in a health care system where such a high completion rate has been achieved. If one judges this project to have had success in recruitment then it can be attributed to the fact that the project was constructed around the clinical needs of GPs and nurses. We provided a patient specific feedback service in a supportive non-judgemental way. We gave practices back more information than they sent us, together with the provision of a telephone helpline. It cannot be over emphasised that this was not a survey or a questionnaire - it was an intensive 'hands on, effort required' clinical audit package.

The participating practices are, by definition, more interested in asthma than those who did not take part. ${ }^{15}$ The results thus have a bias in favour of proactive structured care. The patient sample of 9,467 comprises patients of all ages deemed by their practices to suffer from asthma. Practice asthma registers may contain the names of some young children with wheeze in association with viral respiratory illness whom may or may not turn out to be 'asthmatic'. Similarly, there may be middle aged and elderly patients classified as 'asthma' who may have Chronic Obstructive Airways Disease. The proportion of patients managed at each step of the BTS guidelines is in line with previous work and shows a slow steady rise in the proportion of patients prescribed inhaled steroids. ${ }^{16}$

The patient sample is derived from primary care but contains a wealth of health service utilisation data relevant to both primary and secondary care. In numerical terms, the majority of care is carried out by GPs and practice nurses. Only a small proportion of patients (5\%) were seen in hospital outpatients, with even fewer attending A\&E (3\%) or being admitted (2.5\%). The quiet revolution in the process of asthma care in the UK within the past decade is apparent. Most practices employed a practice nurse with a recognised asthma diploma and the nurse was in turn responsible for carrying out the majority of routine clinical assessments on patients.

This study has several implications. Practices throughout Scotland were willing to participate in a labour intensive audit project based on the use of recommended guidelines. The 'human cost' of asthma remains unacceptably high. The economic impact of asthma is considerable. People with asthma, their health care professionals, and the health service need to work together to reduce the high human and economic burden of asthma in Scotland.

\section{References}

1. ISAAC The International Study of asthma and Allergies in Childhood Steering Committee World wide variations in the prevalence of symptoms of asthma, allergic rhinoconjunctivitis, and atopic eczema. Lancet 1998;351:1225-32.

2. National Asthma Campaign Scotland. Who cares about asthma in Scotland? Report from NAC, 2a North Charlotte Street, Edinburgh.

3. Jones K. Asthma care in General Practice - time for revolution?. Br J Gen Pract 1991;41:224-5.

4. Hoskins G, Neville RG, Smith B, Clark RA. The

link between nurse training and asthma outcomes. $\mathrm{Br}$

J Com Nursing 1999;4:222-8.

5. Grampian Asthma Study of Integrated Care (GRASSIC). A clinical, social and economic evaluation. BMJ 1994;308:559-564.

6. British Thoracic Society et al. British Guidelines on Asthma Management. Thorax 1997;52(Suppl 1):S1S21.

7. Weiss KB, Sullivan SD. The Economic costs of asthma: a review and conceptual model.

Pharmacoeconomics 1993;4:14-30.

8. Neville RG, Hoskins G, Clark RA, McCowan C, Smith B. A standardised recording system to monitor outcomes. The Asthma Journal 2001;6 (4):193-196. 9. McCowan C, Neville RG, Ricketts IW, Warner FC, Hoskins G, Thomas GE. Computer assisted assessment and management of patients with asthma. Am J Resp \& Crit Care Med April 2001;163(5):A506. 10. Centre for Medical Education, University of Dundee. Moving to Audit - An educational package for Nurses, Midwives and Health Visitors. The Postgraduate Office, Ninewells Hospital and Medical School, Dundee, 1994;ISBN:1 871749420.

11. Neville RG, Clark RA, Hoskins G, Smith B for General Practitioners in Asthma Group. National Asthma Attack Audit. BMJ 1993;306:559-562. 12. Neville RG, Hoskins G, Smith B, Clark RA. Observations on the structure, process and clinical outcomes of asthma care in general practice. $\mathrm{Br} J$ Gen Pract 1996;46:583-587.

13. Netten A, Dennett J Unit Costs of Health and Social Care, PSSRU, University of Kent: 1997

14. Hoskins G, McCowan C, Neville R G, Thomas G E, Smith B, Silverman S. Risk factors and costs associated with an asthma attack. Thorax 2000;55(1):19-24.

15. Neville R G, Hoskins G, Smith B. Asthma audit for enthusiasts and sceptics. Asthma in General Practice 1998;6(2):19-22.

16. Neville RG, Pearson MG, Richards N, Patience J, Sondhi S, Wagstaff B, Wells NA Cost analysis on the pattern of asthma prescribing in the UK. ERJ 1999;14:605-609.

\section{Acknowledgements}

We thank all the GPs, practice nurses and patients who participated.

The Project Steering Group comprised Mrs Gaylor Hoskins, Mrs Barbara Smith, Dr Ron Neville, Mr Colin McCowan, Prof Ian Ricketts, Dr Giles Thomas, Dr Harry Leadbetter, Dr Hugh Whyte, Dr Alison McIsaac, Dr James Paton, Dr Christine Bucknall, Dr Roland Clark, Ms Arlene Napier and Ms Marjory Burns.

The project was funded by the Clinical Resource and Audit Group (CRAG) of the Chief Scientists Office of the Scottish Office. 